\title{
VALIDACIÓN ESTRUCTURAL DEL WONG-LAW EMOTIONAL INTELLIGENCE SCALE (WLEIS): ESTUDIO PRELIMINAR EN ADULTOS
}

\section{STRUCTURAL VALIDATION OF THE WONG-LAW EMOTIONAL INTELLIGENCE SCALE (WLEIS): PRELIMINARY STUDY IN ADULTS}

\author{
César Merino Soto*, Manuel Lunahuaná-Rosales ${ }^{\star \star}$ y Rabindra Kumar Pradhan ${ }^{\star \star \star}$ \\ Universidad de San Martín de Porres, Perú \\ Indian Institute of Technology Kharagpur, India
}

\section{RESUMEN}

La medición de la inteligencia emocional ha tenido muchas propuestas en formato de autorreporte. Una de estas es el Wong-Law Emotional Intelligence Scale (WLEIS), creada para el contexto laboral y que evalúa valoración y expresión de las emociones propias, valoración y reconocimiento de las emociones en otros, regulación de las propias emociones y uso de la emoción para facilitar el desempeño. Hay poca evidencia psicométrica sobre el Wong-Law Emotional Intelligence Scale en habla hispana, lo que posiblemente conduce a que sea también poco utilizado en la investigación y práctica profesional. El objetivo del presente estudio es presentar los primeros resultados de la validez de la estructura interna del WLEIS en adultos peruanos. Fueron 120 participantes (72 mujeres) entre 17 y 59 años, quienes respondieron al cuestionario mediados por internet. Se analizó la estructura interna mediante metodología de ecuaciones estructurales. Se halló una satisfactoria estructura de 4 factores y elevadas cargas factoriales de los ítems; las correlaciones interfactores fueron altas o moderadamente altas; y la consistencia interna fue buena. Se concluye que los primeros resultados son aceptables para asumir que el modelo de Wong-Law también es aparentemente generalizable.

Palabras clave: Inteligencia emocional, evaluación, análisis factorial, peruanos.

\begin{abstract}
The measurement of emotional intelligence has had many proposals in the form of self-report. One of these is the Wong-Law Emotional Intelligence Scale (WLEIS), created for the job context and which evaluates valuation and the expression of self-emotions; valuation and recognition of emotions in others; regulation of one's own emotions and the use of emotion to facilitate performance. There is little psychometric evidence regarding the Wong-Law Emotional Intelligence Scale in the Spanish speaking world, which could lead to it also being little used in research and professional practice. The objective of this study is to present the first results of the internal structure validity of the WLEIS in Peruvian adults. There were 120 participants ( 72 women) between 17 and 59 years old who responded to the questionnaire mediated by the internet. The internal structure was analyzed using structural equations methodology. A satisfactory structure of 4 factors and high factorial loads of items was found. The inter-factorial correlations were high or moderately high - and the internal consistency was good. It is concluded that the first results are acceptable - to assume that the Wong-Law model is also apparently generalizable.
\end{abstract}

Keywords: Emotional Intelligence, evaluation, factorial analysis, Peruvians 
Una de las más importantes contribuciones de los psicólogos en la última década del siglo XX ha sido la incorporación del constructo de inteligencia emocional a la comprensión de la conducta organizacional (Pradhan y Mathur, 2008), debido a su influencia sobre el desarrollo de competencias que optimizan el capital humano (Davies, Stankov y Roberts, 1998). La primera definición formal como término de la inteligencia emocional (IE) fue la que realizaron Salovey y Mayer (1990), quienes la definieron como la capacidad para poder monitorear los sentimientos y emociones de uno mismo y de otros, poder distinguirlos y utilizar dicha información con el fin de orientar nuestro pensamiento y actuar. Consecuentemente y con el afán de un mejor estudio de la IE, se formularon dos modelos básicos que Mayer, Salovey y Caruso (2000) describen de forma clara. Por una parte, el modelo de capacidad considera a la IE como una forma cognitiva pura que utiliza las emociones de manera adaptativa en la generación de pensamientos. Por otro lado, el modelo mixto considera a la IE como un conjunto de habilidades cognitivas además de rasgos estables de personalidad, competencias socioemocionales y factores motivacionales (Fernández y Extremera, 2005). Adicionalmente, la IE podía ser medida de acuerdo a dos visiones distintas (Pérez, Petrides, y Furnham, 2005; Petrides y Furnham, 2001). La primera toma de referencia a la IE capacidad, vista como una capacidad cognitiva emocional que debería ser medida a través del test de rendimiento máximo. Por otro lado, se presenta la IE como rasgo, que refiere a la autoeficacia emocional, a las percepciones propias acerca de cómo se reconoce, procesa y se utiliza la información emocional, que debe ser medida a través de cuestionarios de autoinforme.

En base al antes ya mencionado modelo mixto y considerando como forma de medida la IE rasgo, Wong y Law (2002) desarrollaron la Escala de Inteligencia Emocional de Wong y Law (WLEIS) en primera instancia para tener un instrumento breve que se pueda utilizar en investigaciones acerca del liderazgo, administración y rendimiento laboral. Para elaborar esta escala, los autores utilizaron la definición de Davies et al. (1998), quienes expresan que la IE está compuesta por las cuatro siguientes dimensiones: (a) Valoración y expresión de las emociones propias, área que hace referencia a la capacidad individual para comprender y expresar las emociones sin dificultad, (b) Valoración y reconocimiento de las emociones en otros, capacidad para poder reconocer adecuadamente las emociones de las personas a nuestro alrededor, (c) Regulación de las propias emociones, capacidad para modular las emociones, y (d) Uso de la emoción, orientación de las emociones hacia la realización de acciones productivas. Wong y Law (2002) reportaron índices de consistencia interna de .87, .90, .84 y .83, respectivamente, y que pueden considerarse para describir estas cualidades en grupos de personas. Otros estudios también han reportado similares niveles de confiabilidad (p. ej., Kong, Zhao y You, 2012; Pena y Extremera, 2012; Shi y Wang, 2007; Yin, Lee, Zhang y Jin, 2013), lo que sugiere que sus puntajes pueden ser suficientemente precisos para la investigación grupal e incluso descripción individual. Pero estos trabajos de validación realizadas con el WLEIS han predominado en países no hispanos (por ejemplo, Aslan y Erkus, 2008; Shi y Wang, 2007; Fukuda et al., 2011; Rodrigues, Rebelo y Coelho, 2011). Algunos estudios no publicados realizados en España (p. ej., Fernández-Berrocal, Pérez, Repetto y Extremera, 2004; Pérez, Repetto y Echevarría, 2004) y un trabajo en adolescentes (Vila y Pérez-Gonzales, 2007) parecen ser las únicas y primeras fuentes bibliográficas de su validación en habla hispana. Adicionalmente, el WLEIS ha sido positivamente considerado como una medida autopercibida orientada desde el modelo de habilidad (Meisler y Vigoda-Gadot, 2014). Este panorama crea la motivación para ver si la estructura del WLEIS puede ser generalizable en hablantes hispanos, específicamente en el Perú.

En Perú han destacado algunos estudios psicométricos para validar medidas de IE, por ejemplo, los trabajos de validación del Emotional Quotient Inventory (EQ-i; BarOn, 1997) y Emotional Quotient Inventory: Young Version (EQ-i: YV; Bar-On y Parker, 2000) en participantes adultos (Ugarriza, 2001), y en niños (Ugarriza y Pajares, 2005), respectivamente. Por otra parte, algunos de estos resultados parecen dar solo una incompleta, o insatisfactoria evidencia de la validez estructural de estas adaptaciones. Por ejemplo, en el estudio con el EQ-i adultos (Ugarriza, 2001), se pueden observar coeficientes de confiabilidad incluso debajo de .61 en los subcomponentes, sin evaluarse su impacto cuantitativo y cualitativo. También, las correlaciones entre los subcomponentes muestran correlaciones elevadas no solo

* cmerinos@usmp.pe

**manuel_lunahuana1@hotmail.com

***rkpradhan@hss.iitkgp.ernet.in

LIBERABIT: Lima (Perú) 22(1): 103-110, 2016 
en sus respectivas dimensiones de orden superior, sino entre las otras, sugiriendo posiblemente complejidad factorial (Merino y Grimaldo, 2010). Respecto de validación de EQ-i: YV en adolescentes y niños peruanos (Ugarriza y Pajares, 2005), Merino, García y Navarro (2014) también hallaron cuestionamientos para su uso clínico y de investigación debido a la debilidad de los puntajes respecto de sus estimaciones de consistencia interna. Dado el contexto anterior, el objetivo del presente estudio es aportar con la validación preliminar del WLEIS en adultos, dentro de un marco de avance de investigación, además de expandir las opciones de medición de IE mediante el enfoque teórico subyacente al WLEIS. Esta primera evaluación consistió en verificar su estructura interna, orientándola hacia las relaciones internas entre los ítems. Esta evaluación sirve también para verificar la consistencia del marco teórico del WLEIS respecto de sus cuatro componentes conceptuales.

\section{Método}

\section{Participantes}

La muestra para esta investigación estuvo formada por 120 personas seleccionadas de acuerdo a un muestreo intencional, a través de una conocida red social utilizada en el país. Los criterios de exclusión principales fueron que los participantes declaren ser de una nacionalidad distinta a la peruana y la edad, menor a 17 años. Todos los sujetos que llenaron el instrumento fueron seleccionados, pues cumplimentaron los criterios descritos y se obtuvo el 100 $\%$ de tasa de respuesta. Dentro de la muestra, 72 personas son mujeres (60\%), mientras que 48 de ellos son hombres (40\%). Sus edades oscilan entre los 17 y 59 años, con una media de $26.62(D E=8.52)$. La mayor parte de los participantes tuvieron estudios universitarios completos y en proceso (80.9\%).

\section{Instrumentos}

Escala de Inteligencia Emocional de Wong-Law (WLEIS; Wong y Law, 2002). Esta escala es una medida de autoinforme, compuesta por 4 factores y 16 ítems, 4 ítems por cada factor. Usa un formato de respuesta ordinal de 7 puntos (desde 1 = completamente en desacuerdo, hasta 7 = completamente de acuerdo). Las 4 subescalas o factores son (a) valoración y expresión de las emociones propias, (b) valoración y reconocimiento de las emociones en otros, (c) regulación de las propias emociones y (d) uso de la emoción para facilitar el desempeño. En el presente estudio se usó la adaptación realizada en España, en que se efectuó la traducción y adaptación lingüística para un estudio en adolescentes (Vila y Pérez-Gonzáles, 2007). El instrumento presenta el ordenamiento de los ítems de manera no aleatoria y consecutiva para cada uno de los 4 factores. Con respecto a las instrucciones establecidas en el cuestionario, se refiere lo siguiente: «A continuación encontrará algunas afirmaciones sobre sus emociones y sentimientos. Lea atentamente cada frase y señale con una ' $\mathrm{X}$ ' su grado de acuerdo con cada una de las afirmaciones. No hay respuestas correctas o incorrectas, ni buenas o malas. No emplee mucho tiempo en cada respuesta». Los autores en el estudio original (Wong y Law, 2002) refieren que los índices de consistencia interna son desde .83 hasta .90. En España (Vila y Pérez-Gonzales, 2007) también se reportan adecuados índices de confiabilidad.

\section{Procedimiento}

La recolección de datos se hizo por medio de un procedimiento mediado por internet (Hoerger, 2010), desde el cual se elaboró en un documento electrónico anónimo y confidencial con instrucciones detalladas, una ficha sociodemográfica, el WLEIS y otro cuestionario de otro constructo psicológico. El material fue enviado a los participantes registrados en una red social conocida en Perú, mediante un enlace que dirigía al participante al material construido en Formularios de Google, una aplicación creada para diseñar y enviar encuestas por internet. Antes de responder los cuestionarios, los que participaron dentro del mismo formulario respondieron en una hoja de participación voluntaria, que fue condicional para continuar con la encuesta. No se programó algún tiempo límite para el llenado, y las instrucciones generales enfatizaron el anonimato, la honestidad de la respuesta y la atención a las instrucciones de cada cuestionario. Una vez completados los cuestionarios, se hizo un escrutinio para asegurar la calidad de los datos, evaluando patrones de respuesta. El análisis consistió en aplicar un enfoque de análisis factorial semiconfirmatorio mediante una rotación procusteana semiespecificada (Browne, 1972), en que se establecen a priori las características estructurales (ubicación o magnitud) de las cargas factoriales de los

* cmerinos@usmp.pe

**manuel_lunahuana1@hotmail.com

***rkpradhan@hss.iitkgp.ernet.in

LIBERABIT: Lima (Perú) 22(1): 103-110, 2016 
ítems en sus supuestos constructos (cargas convergentes) y en sus constructos no correspondientes (cargas divergentes). Este método puede ser apropiado para verificar una estructura factorial sin imponer las restricciones del análisis factorial confirmatoriomodelamiento de ecuaciones estructurales, considerando la fuerza de la teoría y la racionalidad de estas restricciones en instrumentos orientadas a la personalidad. Para el presente estudio, las cargas convergentes se estimaron libremente y las cargas factoriales divergentes se fijaron en .25 (debido a que la esperada correlación entre las variables latentes produciría al menos moderada complejidad factorial). La fuerza de las cargas divergentes se examinaron mediante la obtención de coeficientes estructurales (Graham, Guthrie y Thomson, 2003). No se aplicaron otras restricciones para evaluar el modelo de medición del WLEIS. La medida principal de ajuste de los ítems, los factores y de la solución total fue obtenida mediante el coeficiente de congruencia (Tucker, 1951), una medida usual para el análisis del ajuste de soluciones factoriales (Lorenzo-Seva y ten Berge, 2006). El método de extracción fue Unweighted Least Squares (ULS) sobre la matriz de correlaciones Pearson interítems. Todo el procedimiento analítico se hizo mediante el programa Factor (Lorenzo-Seva y Ferrando, 2006).

\section{Resultados}

Respecto al análisis factorial (Tabla 1), se halló en general que la congruencia de los ítems, factores y solución total estuvo alrededor o fue mayor al criterio mínimo de .85 (Lorenzo-Seva y ten Berge, 2006), y 10 ítems mostraron congruencia mayor a .90. Las cargas factoriales convergentes fueron predominantemente más fuertes que las cargas divergentes, aunque se detectó un ítem con baja validez convergente en SEA (ítem 13). También se detectó una tendencia a la complejidad factorial en varios ítems, pues las cargas estructurales fueron relativamente mayores a .30. La correlación latente interfactorial ocurrió entre moderada y trivial, pero predominante trivial. En contraste, las correlaciones entre las variables observables (puntajes), presentadas en la diagonal superior de la Tabla 1 (sección correlaciones), fueron todas elevadas.

La confiabilidad estimada por el coeficiente $\alpha$ fue altamente satisfactoria en todas las subescalas, pues alcanzaron magnitudes superiores a .85. Los intervalos de confianza al $95 \%$ (Bonett, 2002) para este parámetro en SEA (.812, .89), OEA (.799, .88), UEO (.839, .911) y ROE (.879, .933) también superaron casi completamente el valor .80 , indicando que, poblacionalmente, este parámetro puede también ser aceptable. 
Tabla 1

Estadísticos descriptivos y resultados factoriales

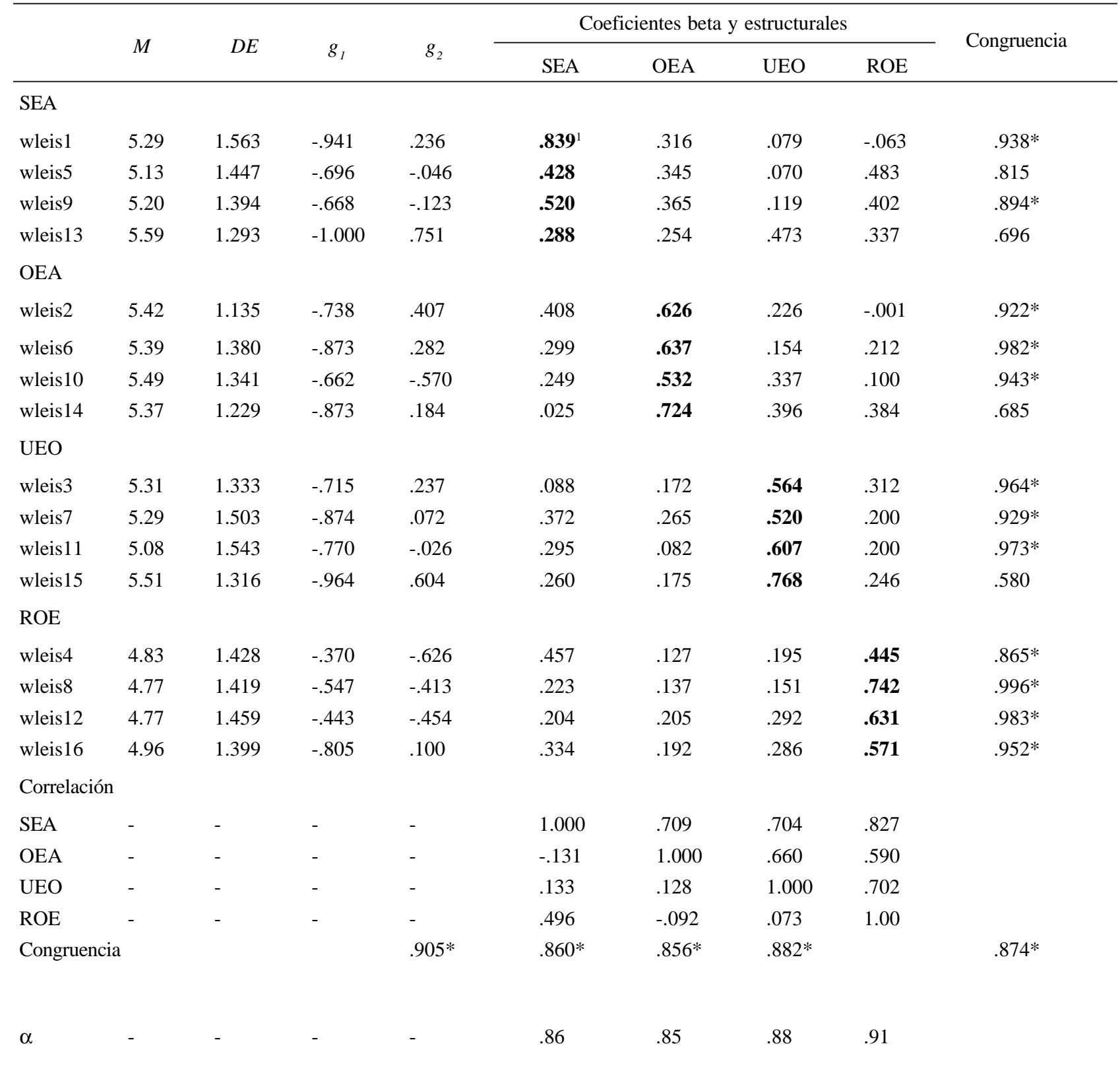

Correlaciones: debajo de la diagonal, correlaciones latentes; arriba de la diagonal, correlaciones entre puntajes. SEA: valoración de propias emociones. OEA: valoración de las emociones de otros. UEO: uso de propias emociones. ROE: regulación de emociones. $g_{1}$ : asimetría de Fisher. $g_{2}$ : curtosis de Fisher. *Congruencia mayor a $0.85 .{ }^{1}$ En negrita, coeficientes beta; sin negrita, coeficientes estructurales.

* cmerinos@usmp.pe

**manuel_lunahuana1@hotmail.com

***rkpradhan@hss.iitkgp.ernet.in

ISSN: 1729-4827 (Impresa)

LIBERABIT: Lima (Perú) 22(1): 103-110, 2016

ISSN: 2233-7666 (Digital) 


\section{Discusión}

El breve estudio realizado presenta los primeros resultados de la validación del WLEIS en Latinoamérica, en una muestra heterogénea de adultos, cuyos datos fueron mediados por internet. En general, se halló una congruencia satisfactoria entre el modelo propuesto (cuatro constructos relacionados) y los datos; por lo tanto, se puede concluir que el modelo de medición de IE propuesto por medio del WLEIS es satisfactorio en esta primera evaluación de su estructura latente. La consistencia interna fue igualmente elevada y satisfactoria, considerando aún la brevedad del número de ítems en cada subescala. Por otro lado, las correlaciones latentes interfactoriales indicaron la relativa independencia de los constructos evaluados por el WLEIS. Este resultado se puede comprender como un modelo definido principalmente de constructos cercanamente ortogonales, en que las correlaciones lineales son bajas entre ellas. Sin embargo, esto no converge con otros estudios de evaluación estructural del WLEIS, pues generalmente se hallan modelos con un factor de segundo orden (Whitman, Van Rooy, Viswesvaran y Kraus, 2009). Pero las correlaciones obtenidas en el presente estudio fueron estimadas usando la especificación de cargas divergentes moderadamente bajas, lo que posiblemente está enmascarando correlaciones de error entre los ítems (Wesfall, Henning y Howell, 2012) que no fueron modelados en el presente estudio. En contraste, las correlaciones entre los puntajes (variables observables) fueron elevadas $(>.50)$, tal como cercanamente se halla en la literatura (Joseph y Newman, 2010; Li, Saklofske, Bowden, Yan y Fung, 2012; Ng, Wang, Zalaquett y Bodenhorn, 2007; Whitman et al., 2009; Wong y Law, 2002). Esta discrepancia parece provenir del impacto de los errores correlacionados de los ítems sobre las correlaciones entre los puntajes (variables observadas), produciendo sobreestimación de estas relaciones (Wesfall et al., 2012). El impacto de los errores correlacionados sobre la confiabilidad y el modelamiento en el marco de factores comunes ha sido previamente definido como problemático (Green y Hershberger, 2000; Komaroff, 1997; Lucke, 2005; Raykov, 1998; Zimmerman y Williams, 1980) y se requiere una evaluación sobre esto en los siguientes estudios con el WLEIS.
Hay aspectos de la validez interna (como la posible presencia de errores correlacionados, la confiabilidad basada en el modelo factorial, invarianza de medición y modelo con factores de segundo orden) y externa (correlatos con constructos de funcionamiento conductual positivo y negativo) que no han sido evaluados, así como la evaluación del modelo de confiabilidad más apropiado desde el marco de la teoría clásica de los test (p. ej., tauequivalente). Este último tiene un impacto serio sobre la magnitud del coeficiente (Cronbach, 1951; Merino et al., 2014). Estas son cuestiones abiertas a investigación que use una muestra de sujetos de mayor tamaño para garantizar la estabilidad de los resultados. Finalmente, tomando en cuenta los resultados obtenidos en el presente estudio, se ha obtenido una satisfactoria validación que sirve para plantear objetivos específicos de trabajo para la verificación en una investigación que replique las características estructurales del WLEIS, así como sus correlatos con criterios conductuales.

\section{Referencias}

Aslan, S. \& Erkus, A. (2008). Measurement of emotional intelligence: Validity and reliability studies of two scales. World Applied Science Journal, 4(3), 430-438.

Bar-On, R. (1997). The Bar-On Emotional Quotient Inventory (EQ-i): A Test of Emotional Intelligen-ce. Toronto, Canadá: Multi-Health Systems.

Bar-On, R. \& Parker, J. D. A. (2000). The Bar-On Emotional Quotient Inventory: Youth Version (EQ-i: YV). Toronto, Canadá: Multi-Health Systems

Bonett, D. G. (2002). Sample size requirements for testing and estimating coefficient Alpha. Journal of Educational and Behavioral Statistics, 27(4), 335-340.

Browne, M. (1972). Oblique rotation to a partially specified target. British Journal of Mathematical and Statistical Psychology, 25(2), 207-212.

Cronbach, L. J. (1951). Coefficient alpha and the internal structure of tests. Psychometrika, 16(3), 297-334.

Davies, M., Stankov, L., \& Roberts, R. D. (1998). Emotional intelligence: In search of an elusive construct. Journal of Personality and Social Psychology, 75(4), 989-1015.

Fernández, P. \& Extremera, N. (2005). La inteligencia emocional y la educación de las emociones desde el modelo de Mayer y Salovey. Revista Interuniversitaria de Formación del Profesorado, 19(3), 63-93. 
Fernández-Berrocal, P., Pérez, J. C., Repetto, E. \& Extremera, N. (2004). Una comparación empírica entre cinco medidas breves de inteligencia emocional percibida. En Proceedings of VII European Conference on Psychological Assessment. Málaga: European Association of Psychological Assessment.

Fukuda, E., Saklofske, D. H., Tamaoka, K., Fung, T., Miyaoka, Y., \& Kiyama, S. (2011). Factor structure of Japanese versions of two emotional intelligence scales. International Journal of Testing, 11, 71-92.

Graham, J. M., Guthrie, A. C., \& Thompson, B. (2003). Consequences of not interpreting structure coefficients in published CFA research: A reminder. Structural Equation Modeling, 10(1), 142-153.

Green, S. B. \& Hershberger, S. L. (2000). Correlated errors in true score models and their effect on coefficient alpha. Structural Equation Modeling, 7(2), 251-270.

Hoerger, M. (2010). Participant dropout as a function of survey length in internet mediated university studies: Implications for study design and voluntary participation in psychological research. Cyberpsychology, Behavior and Social Networking, 13(6), 697-700.

Joseph, D. L. \& Newman, D. A. (2010). Discriminant validity of self-reported emotional intelligence: a multitraitmultisource study. Educational and Psychological Measurement, 70(4), 672-694.

Komaroff, E. (1997). Effect of simultaneous violations of essential tau equivalence and uncorrelated error on coefficient alpha. Applied Psychological Measurement, 21(4), 337-348.

Kong, F., Zhao, J., \& You, X. (2012). Emotional intelligence and life satisfaction in Chinese university students: The mediating role of self-esteem and social support. Personality and Individual Differences, 53(8), 1039-1043.

Li, T., Saklofske, D. H., Bowden, S. C., Yan, G., \& Fung, T. S. (2012). The measurement invariance of the Wong and Law Emotional Intelligence Scale (WLEIS) across three Chinese university student groups From Canada and China. Journal of Psychoeducational Assessment, 30(4) 439-452.

Lorenzo-Seva, U. \& Ferrando, P. J. (2006). FACTOR: A computer program to fit the exploratory factor analysis model. Behavioral Research Methods, Instruments and Computers, 38(1), 88-91.

Lorenzo-Seva, U. \& ten Berge, J. M. F. (2006). Tucker's congruence coefficient as a meaningful index of factor similarity. Methodology: European Journal of Research Methods for the Behavioral and Social Sciences, 2(2), 5764.

Lucke, J. F. (2005). «Rassling the hog»: The influence of correlated item error on internal consistency, classical reliability, and congeneric reliability. Applied Psychological Measurement, 29(2), 106-125.
Mayer, J. D., Salovey, P., \& Caruso, D. R. (2000). Models of emotional intelligence, En R. J. Sternberg (Ed.), Handbook of intelligence (pp. 396-420). Cambridge: Cambridge University Press.

Meisler, G. \& Vigoda-Gadot, E. (2014). Perceived organizational politics, emotional intelligence and work outcomes. Personnel Review, 43(1), 116-135.

Merino, C. \& Grimaldo, M. (2010). Complejidad factorial de la permisividad moral hacia las conductas morales controvertidas. Interdisciplinaria, 27(2), 297-313.

Merino, C., García, W. \& Navarro, J. (2014). Evaluación de la confiabilidad del Inventario de Inteligencia Emocional de BarOn ICE: NA. Revista Peruana de Psicología y Trabajo Social, 3(1), 141-154.

Ng, K. M., Wang, C., Zalaquett, C. P., \& Bodenhorn, N. (2007). A confirmatory factor analysis of the Wong and Law Emotional Intelligence Scale in a sample of international college students. International Journal for the Advancement of Counselling, 30, 131-144.

Pena, M. \& Extremera, N. (2012). Perceived emotional intelligence in primary school teachers and its relation with the levels of burnout and engagement. Revista de Educación, 359, 604627.

Pérez, J. C., Petrides, K. V., \& Furnham, A. (2005). Measuring trait emotional intelligence. In R. Schulze and R. D. Roberts (Eds.), International Handbook of Emotional Intelligence. Cambridge, MA: Hogrefe\& Huber.

Pérez, J. C., Repetto, E., \& Echeverría, E. (2004). Adaptación al español de las escalas de inteligencia emocional de Wong y Law. Trabajo de investigación sin publicar. Dpto. MIDE II, Facultad de Educación, UNED.

Petrides, K. V. \& Furnham, A. (2001). Trait emotional intelligence: Psycometric investigation with reference to established trait taxonomies. European Journal of Personality, 15(6), 425-448.

Pradhan, R. K. \& Mathur, P. (2008). Emotional Intelligence: Perspectives in Organizations. Delhi: Academic Excellence Publishers.

Raykov, T. (1998). Coefficient alpha and composite reliability with interrelated nonhomogeneous items. Applied Psychological Measurement, 22(4), 375-385.

Rodrigues, N., Rebelo, T., \& Coelho, J. (2011). Adaptação da Escala de Inteligência Emocional de Wong e Law (WLEIS) e análise da sua estrutura factorial e fiabilidade numa amostra portuguesa. Psychologica, 55, 189-207.

Salovey, P. \& Mayer, J. D. (1990). Emotional intelligence. Imagination, Cognition and Personality, 9(3), 185-211.

Shi, J. \& Wang, L. (2007). Validation of emotional intelligence scale in Chinese university students. Personality and Individual Differences, 43(2), 377-387. 
Tucker, L. R. (1951). A method for synthesis of factor analysis studies. Personnel Research Section Report, 984. Washington, D. C.: Department of the Army.

Ugarriza, N. (2001). La evaluación de la inteligencia emocional a través del inventario de BarOn (I-CE) en una muestra de Lima Metropolitana. Personas, 4, 129-160.

Ugarriza, N. \& Pajares, L. (2005). La evaluación de la inteligencia emocional a través del Inventario de Bar-On ICE: NA, en una muestra de niños y adolescentes. Personas, 8, 11-58.

Vila, E. \& Pérez-González, J. C. (2007). Madurez para la carrera e inteligencia emocional en alumnado de secundaria: una aproximación correlacional. En F. Etxeberría, L. Sarasola, J. F. Lukas, J. Etxeberría, \& A. Martxueta (coords.), XIII Congreso Nacional de Modelos de Investigación Educativa (pp. 712-717). Donostia: Erein/AIDIPE.

Wesfall, P. H., Henning, K. S., \& Howell, R. D. (2012). The Effect of error correlation on interfactor correlation in psychometric measurement. Structural Equation Modeling: A Multidisciplinary Journal, 19(1), 99-117.
Whitman, D. S., Van Rooy, D. L., Viswesvaran, C., \& Kraus, E. (2009). Testing the second-order factor structure and measurement equivalence of the Wong and Law emotional intelligence scale across gender and ethnicity. Educational and Psychological Measurement, 69(6), 1059-1074.

Wong, C. S. \& Law, K. S. (2002). The effects of leader and follower emotional intelligence on performance and attitude: An exploratory study. Leadership Quarterly, 13(3), 243-274.

Yin, H., Lee, J., Zhang, Z., \& Jin, Y. (2013). Exploring the relationship among teachers' emotional intelligence, emotional labor strategies and teaching satisfaction. Teaching and Teacher Education, 35, 137-145.

Zimmerman, D. W. \& Williams, R. H. (1980). Is classical test theory «robust» under violation of the assumption of uncorrelated errors? Canadian Journal of Psychology, 34(3), 227-237.

\footnotetext{
*, ** Universidad de San Martín de Porres, Perú.

*** Indian Institute of Technology Kharagpur, India.
} 